S.D. Ryder, D.J. Pisano, M.A. Walker, and K.C. Freeman, eds.

\title{
Mass Determination in the Late-Type Spiral NGC 5457
}

\author{
Ivânio Puerari \\ INAOE, Calle Luis Enrique Erro \#1, 72840 Tonantzintla, Pue, México \\ Margarita Valdez-Gutiérrez \\ Departamento de Astronomía, Universidad de Guanajuato, Apartado \\ Postal 144, 36000 Guanajuato, Gto, México \\ Margarita Rosado \\ IA-UNAM, Apartado Postal 70-264, Ciudad Universitaria, 04510 \\ México D.F., México
}

\begin{abstract}
We have performed a kinematical and dynamical analysis of the ionized gas content of the late-type spiral NGC 5457 (M101) by means of scanning Fabry-Perot interferometry. The purpose of this study is to gain insight into the dark matter content in this galaxy. A sample of 263 HII regions were catalogued. We calculated radial velocity and velocity dispersions. From the dynamics of the ionized gas we were able to infer a mass of $9.8 \times 10^{10} \mathrm{M}_{\odot}$ inside a radius of 4.8 (or $10 \mathrm{kpc}$ ).
\end{abstract}

The nearly face--on giant Scd spiral galaxy NGC 5457 (M101) has been adopted - by far - as a standard candle in numerous galactic studies. The interest is reflected in multiwavelength analyses focused on its stellar (Bresolin et al. 1998, Willick \& Batra 2001, and references therein) and gaseous contents (Waller et al. 1997). Regarding the behaviour of the ionized gas, it has been thoughtfully investigated ranging from long-slit to Fabry-Perot interferometers. Comte et al. (1979) performed the first FP study using an image-tube intensifier, reporting the dynamics of NGC 5457.

In this contribution we present recent results derived from a Fabry-Perot study. We have used the UNAM Scanning Fabry-Perot interferometer PUMA attached to the f/7.9 Ritchey-Chrètien focus of the $2.1 \mathrm{~m}$ telescope. The PUMA setup is composed of a scanning Fabry-Perot interferometer, a focal reducer with a f/3.95 camera, a filter wheel, a calibration system and a SITE3 CCD detector $(1024 \times 1024$ pixels $)$. For other main characteristics of the instrumental setup, see Rosado et al. (1995) as well as Table 2 in Valdez-Gutiérrez et al. (2001). FabryPerot reductions (phase calibration and basic kinematical analysis) were carried out using the software package AdHoc (Boulesteix 1993). The radial velocity profiles were analysed under a set of FORTRAN programs developed by one of us. These programs fit Gaussian functions once the profile is quadratically deconvolved of the instrumental function (an Airy function).

The histogram of radial velocity dispersion $\left(\sigma_{R}\right)$ using the sample of 263 HII regions presents a normal distribution with mean $\left\langle\sigma_{R}\right\rangle=30.02 \mathrm{~km} \mathrm{~s}^{-1}$ 
(supersonic dispersion) and $\sigma_{<\sigma_{R}>}=3.42 \mathrm{~km} \mathrm{~s}^{-1}$. This value is in agreement with that calculated by Melnick (1977) for some HII regions of M101.

We calculate the rotation curve and fit a disk + halo model using the equations from Borriello \& Salucci (2001) (see Fig. 1, left). A pure disk model was also used (Fig. 1, right). The total mass inside a radius of 4.8 (or $10 \mathrm{kpc}$ ) is $9.8 \times 10^{10} M_{\odot}$, in agreement with Comte et al. (1979). It is evident in these figures that the halo contribution within the central $10 \mathrm{kpc}$ is very small (see also Comte et al. 1979). Furthermore, an important feature can be seen in the innermost part. The step raising of the rotation curve can only be explained by a central mass concentration. We are working on some models to quantify the amount of this mass and the results will be published elsewhere.
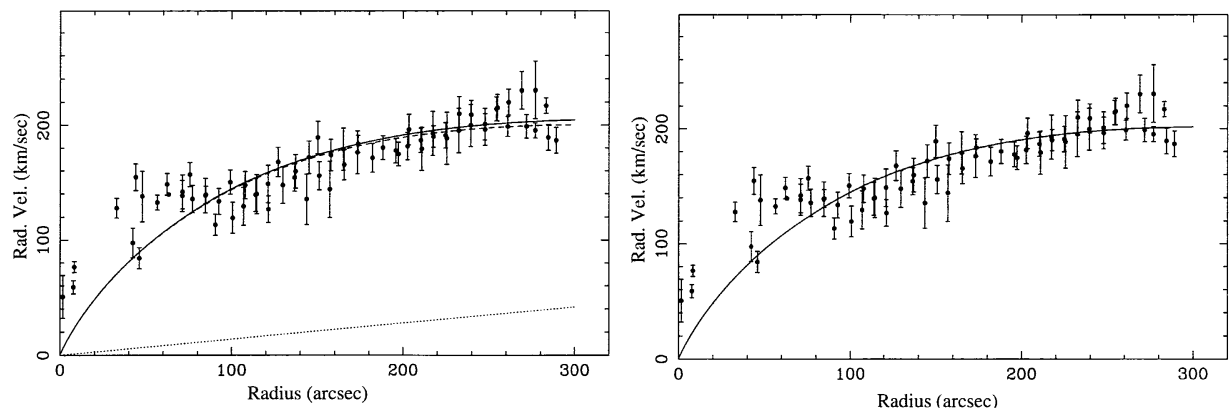

Figure 1. $\mathrm{H} \alpha$ Rotation curve with disk + halo (left) and pure disk (right) fits.

Acknowledgments. IP and MVG thank the Mount Stromlo Observatory for its hospitality and financial support during their sabbatical and visitor stays, respectively. This work is also supported by the Mexican Foundation CONACyT under project No. 36078-E.

\section{References}

Borriello, A., Salucci, P. 2001, MNRAS, 323, 285

Boulesteix, J. 1993, "ADHOC Reference Manual", Publications de l'Observatoire de Marseille (http://alpha2.cnrs-mrs.fr/adhoc/)

Bresolin, F., Kennicutt, R. C., Ferrarese, L. et al. 1998, AJ, 116, 119

Comte, G., Monnet, G., Rosado, M. 1979, A\&A, 72, 73

Melnick, J. 1977, ApJ, 213, 15

Rosado, M. et al. 1995, Rev. Mex. Astron. y Astrof., 3, 263

Valdez-Gutiérrez, M. et al. 2001, A\&A, 366, 35

Waller, W. H., Bohlin, R. C., Cornett, R. H. et al. 1997, ApJ, 481, 169

Willick, J. A., Batra, P. 2001, ApJ, 548, 564 